\title{
Novel mutation in NLRP3 Exon 7 results in sensorineural hearing loss without chronic inflammation
}

\author{
L Broderick ${ }^{1,2^{*}}$, S Cherukumilli Grevich ${ }^{3}$, C Putnam $^{1,4}$, H Hoffman $^{1,2}$ \\ From 8th International Congress of Familial Mediterranean Fever and Systemic Autoinflammatory Diseases \\ Dresden, Germany. 30 September - 3 October 2015
}

\section{Introduction}

Cryopyrin-associated periodic syndromes (CAPS) are a spectrum of autoinflammatory disesase with spontaneous activation of the NLRP3 inflammasome, leading to hypersecretion of IL-1b. To date, more than 90 mutations have been described in NLRP3,primarily in exon 3, leading to the autoinflammatory symptoms observed in CAPS. Here, we describe a family with autosomal dominantly inherited, unilateral sensorineural hearing loss, found to have a novel mutation in exon 7 of NLRP3.

\section{Objective}

To evaluate the clinical and genetic features of a 2-generation pedigree with unilateral sensorineural hearing loss.

\section{Methods}

Patient data, including audiologic studies, and detailed family history was obtained. DNA was isolated using saliva samples from all family members and Sanger sequencing of all 9 exons of NLRP3 was performed.

\section{Results}

The proband was first identified during an evaluation for recurrent fevers and aphthous stomatitis, occurring 2-3 times per month, beginning at 4 months of age, without infectious etiology. Episodes were not associated with cold exposures or rash. Physical exam was notable for an otherwise well appearing child, without evidence for inflammation or autoantibodies. At the age of 5, he was noted to have unilateral sensorineural hearing loss in the range of $4000-8000 \mathrm{~Hz}$, similar to his father. Two younger siblings were noted to have similar symptoms, with similar age of onset, including unilateral hearing loss. Between flares, ESR and high sensitivity CRP were normal in all three children suggesting an absence of chronic inflammation. We identified a novel variant in NLRP3 exon 7: c.2753 G>A, R918Q in all 4 affected members of this 5-person pedigree. Modeling of the mutation localizes the substituted amino acid residue to the inner surface of the LRR domain and suggests an alteration in charge distribution predicted to affect inter or intraprotein protein-protein interactions.

\section{Conclusions}

While bilateral sensorineural hearing loss has been described in association with Muckle Wells Syndrome and Neonatal onset multi-inflammatory disease (NOMID), both on the CAPS spectrum, in this family, a novel NLRP3 variant was associated with unilateral hearing loss in the absence of serologic evidence for chronic inflammation. This is the first potentially disease associated variant to be described in exon 7 of NLRP3.

\section{Authors' details}

${ }^{1}$ University of California, San Diego, La Jolla, USA. ${ }^{2}$ Rady Children's Hospital, San Diego, San Diego, USA. ${ }^{3}$ University of Washington, Seattle, USA. ${ }^{4}$ Ludwig Institute of Cancer Research, La Jolla, USA.

Published: 28 September 2015

doi:10.1186/1546-0096-13-S1-037

Cite this article as: Broderick et al:: Novel mutation in NLRP3 Exon 7 results in sensorineural hearing loss without chronic inflammation.

Pediatric Rheumatology 2015 13(Suppl 1):O37.

${ }^{1}$ University of California, San Diego, La Jolla, USA

Full list of author information is available at the end of the article 